\title{
Investigating the role of exogenous cueing on selection history formation
}

\author{
Michael A. Grubb ${ }^{1} \cdot$ Gabriela Christensen ${ }^{1} \cdot$ John Albanese ${ }^{1}$
}

Published online: 28 March 2019

(C) The Psychonomic Society, Inc. 2019

\begin{abstract}
An abundance of recent empirical data suggest that repeatedly allocating visual attention to task-relevant and/or rewardpredicting features in the visual world engenders an attentional bias for these frequently attended stimuli, even when they become task irrelevant and no longer predict reward. In short, attentional selection in the past hinders voluntary control of attention in the present. But do such enduring attentional biases rely on a history of voluntary, goal-directed attentional selection, or can they be generated through involuntary, effortless attentional allocation? An abrupt visual onset triggers such a reflexive allocation of covert spatial attention to its location in the visual field, automatically modulating numerous aspects of visual perception. In this Registered Report, we asked whether a selection history that has been reflexively and involuntarily derived (i.e., through abruptonset cueing) also interferes with goal-directed attentional control, even in the complete absence of exogenous cues. To build spatially distinct histories of exogenous selection, we presented abrupt-onset cues twice as often at one of two task locations, and as expected, these cues reflexively modulated visual processing: task accuracy increased, and response times (RTs) decreased, when the cue appeared near the target's location, relative to that of the distractor. Upon removal of these cues, however, we found no evidence that exogenous selection history modulated task performance: task accuracy and RTs at the previously most-cued and previously least-cued sides were statistically indistinguishable. Thus, unlike voluntarily directed attention, involuntary attentional allocation may not be sufficient to engender historically contingent selection biases.
\end{abstract}

Keywords Spatial attention $\cdot$ Selection history $\cdot$ Exogenous attention

\section{Introduction}

From smartphone notifications to motion-filled advertisements in digital newspapers, abrupt onsets are ubiquitous in modern life. Competition for attention is fierce, yet abruptonset stimuli do a remarkable job of prompting the visual system to bias, albeit briefly, the processing of visual information emanating from a particular location. This automatic response is generated involuntarily, effortlessly, without eye movements, and even when voluntary attention is engaged elsewhere (Grubb, White, Heeger, \& Carrasco, 2015).

Electronic supplementary material The online version of this article (https://doi.org/10.3758/s13423-019-01591-z) contains supplementary material, which is available to authorized users.

Michael A. Grubb

michael.grubb@trincoll.edu

1 Department of Psychology and Neuroscience Program, Trinity College, 300 Summit St, Hartford, CT 06106, USA
Known to attention scientists as covert exogenous spatial attention, this phenomenon of reflexive, selective prioritization has been of widespread empirical interest, and changes in information processing speed, perceptual discriminability, and subjective appearance at exogenously attended locations have been extensively documented in the literature (Cameron, Tai, \& Carrasco, 2002; Carrasco, Ling, \& Read, 2004; Carrasco \& McElree, 2001; Giordano, McElree, \& Carrasco, 2009; Grubb, Behrmann, Egan, Minshew, Heeger, \& Carrasco, 2013; Herrmann, Montaser- Kouhsari, Carrasco, \& Heeger, 2010; Hopfinger \& Mangun, 1998; Liu, Pestilli, \& Carrasco, 2005; Yeshurun \& Carrasco, 1998). That abrupt, peripheral onsets have a rapid, transient, and involuntary impact on perception is a defining characteristic of covert exogenous attention: behavioral effects peak $\sim 100 \mathrm{~ms}$ after cue onset and decay rapidly thereafter, standing in stark contrast to covert endogenous attention, which takes longer to be voluntarily deployed $(\sim 300 \mathrm{~ms})$ and can be sustained with cognitive effort (Ling \& Carrasco, 2006; Muller \& Rabbitt, 1989; Nakayama \& Mackeben, 1989). 
Recent research concerning the impact of an observer's attentional history on her present attentional state (i.e., attentional selection history; Awh, Belopolsky, \& Theeuwes, 2012) has challenged a long-standing conceptualization of attentional control as being either top-down (i.e., voluntary, endogenous, goal-driven) or bottom-up (i.e., involuntary, exogenous, stimulus-driven). Repeatedly allocating goal-directed attention to a non-salient stimulus feature (e.g., a specific color in a multicolor array) has been shown to engender an attentional selection bias for the repeatedly attended feature, even when that feature becomes task irrelevant, and even when attending to it conflicts with current goals (e.g., Grubb \& Li, 2018). Persistent and reflexive attentional selection biases for objects or features that once signaled reward have also been extensively documented in the literature (Le Pelley, Mitchell, Beesley, George, \& Willis, 2016), thus firmly establishing the notion that physically non-salient stimuli can reflexively draw attention if, through a history of goal-directed attentional selection, proper associations are formed.

Little is known, however, about to what extent effortful, voluntary attention is a necessary component in the formation of such enduring, reflexive selection biases. Do task-irrelevant, abrupt onsets, which reflexively draw attention to a particular location, engender an attentional selection bias for this involuntarily attended location, one that persists in the absence of an explicit exogenous cue?

In this Registered Report (see Attention, Perception, \& Psychophysics, 2013, for a description of and rationale for this publication mechanism), we proposed to test the hypothesis that a history of exogenous selection is capable of generating the kinds of enduring, reflexive attentional biases that have been observed with a history of endogenous selection. Evidence in support of this hypothesis would require a significant reconceptualization of the transient impact of abruptonset stimuli on visual processing specifically, and on voluntary attentional control more generally. A lack of evidence for this hypothesis would suggest that exogenous attentional selection might not be sufficient to impact future attentional control and would indicate that more research is needed to refine our understanding of attentional selection history effects and their causes.

To test our hypothesis, we relied on the wellestablished finding that task-irrelevant, abrupt onsets reflexively modulate visual processing (for a review, see Carrasco, 2011). Two grating stimuli - each rotated clockwise $(\mathrm{CW})$ or counterclockwise $(\mathrm{CCW})$ of vertical - were simultaneously presented in the visual periphery, to the left and right of fixation (Fig. 1, left stream); one was designated as the target, the other as the distractor, and observers reported the orientation of the target grating (two-alternative forced-choice, $\mathrm{CW}$ or $\mathrm{CCW}$ of vertical). We manipulated covert exogenous spatial attention by briefly presenting an abrupt-onset cue near the location of the forthcoming target on half of the trials (valid cue) and near the location of the forthcoming distractor on the other half of the trials (invalid cue).

To evaluate the possibility that reflexive attentional selection engenders a bias for involuntarily attended locations, we introduced a novel modification to the standard cueing procedure. By presenting the cues more often on one side than the other during the first half of Session 1 (2:1 ratio, most-cued vs. least-cued sides counterbalanced across observers), we generated different selection histories for left and right task locations, and in the second half of the session (Fig. 1, right stream) we eliminated abrupt onset cues entirely. If a history of involuntary attentional selection generates persistent biases, then we should see improved performance accuracy and/or faster response times on the previously most-cued side, relative to the previously leastcued side. To evaluate the persistence of any observed effects, participants returned to the lab 1 day (Session 2) and 1 week (Session 3) after Session 1 to complete an additional 360 trials with no exogenous cues (Fig. 1, right stream).

While it has been established that statistical learning can implicitly guide the allocation of spatial attention (for a review, see Jiang, 2017), it is important to stress that our design is not confounded by such learning insofar as the target and distractor locations are concerned. In both halves of Session 1, the target appeared equally often on both sides of fixation, thus equalizing the prior probability that the target would appear on the left and the right. Furthermore, when cues were presented (i.e., in the first half of Session 1), cue validity was $50 \%$ (equal number of valid and invalid cues), so the cue did not provide any information about the forthcoming target's location either. Only the location of the cue was asymmetrically distributed, which provided no taskrelevant information. ${ }^{1}$ Participants were explicitly informed: (1) that they may see brief flashes (i.e., abrupt onset cues) on some trials, (2) that these flashes would not provide any information about where the target would appear, and (3) that they should do their best to ignore them.

Below, we present and discuss the outcome of this preregistered study. Except where explicitly noted, data were collected and analyzed in accordance with our pre-peerreviewed plan.

\footnotetext{
${ }^{1}$ If the plausibility of this scenario is not intuitive, imagine the extreme case where the cue always appears on the right, but is valid and invalid equally often. By definition, the target must appear on the right when the cue is valid and on the left when the cue is invalid. Thus, the cue provides no spatial information about the forthcoming target's location, which occurs equally often on the right and left sides.
} 


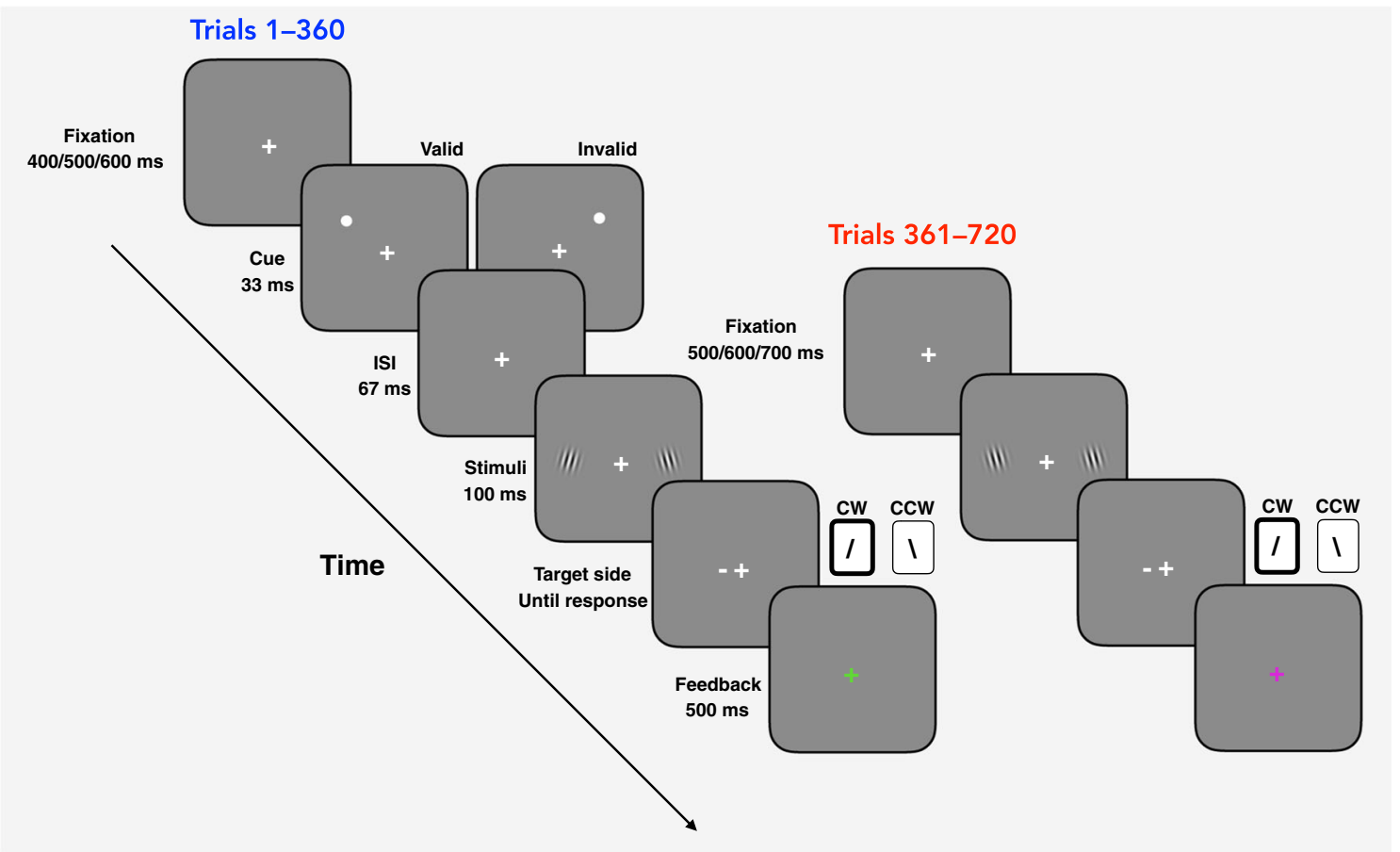

Fig. 1. Trial sequence for Session 1; see text for details

\section{Methods}

\section{Observers}

Forty participants were enrolled in the study and completed at least one experimental session (Session 1). Twenty-two participants were included in the preregistered analyses (mean age $=18.72$ years; self-reported genders: four male, $18 \mathrm{fe}$ male); these participants performed above chance in the task and had sufficient eye-tracking data for necessary control analyses. An additional nine participants were added for the unregistered analyses that do not rely on eye-tracking data (mean age $=19.87$ years; self-reported genders: five male, 26 female), all of whom performed above chance in the task. See Data exclusion criteria below for more details.

Experimental procedures were approved by the Institutional Review Board at Trinity College, and all observers provided written informed consent.

\section{Experimental sessions}

After watching a brief instructional video at the beginning of Session 1, observers completed 48 practice trials, then an orientation discrimination pretest, and lastly the 720 experimental trials. Participants returned to the lab 1 day (Session 2) and 1 week (Session 3) after Session 1, and in each session, completed an additional 360 trials of the orientation discrimination task from Session 1 with no abrupt-onset cues. Observers were compensated US $\$ 10$ per session plus a US $\$ 10$ bonus payment for completing all sessions.

\section{Orientation discrimination thresholds}

The extent to which the grating stimuli were rotated from vertical was determined in a pre-test and then updated after each experimental block, separately for each observer. A 1-up2-down staircase procedure (96 trials, no attentional cues) determined the amount of rotation used in the first block. The amount of rotation was then updated at the end of each experimental block in the following way: overall percent correct was calculated for all trials in the block, the difference between this value and $75 \%$ was determined, and the rotation was updated by that amount. These adjustments help keep performance accuracy in a range where the accuracy-based effects of attention are observable (i.e., not at floor or ceiling).

\section{Data exclusion criteria}

Nine observers performed below chance in Session 1 and were excluded from all reported analyses (proportion correct range, [0.4778-0.5264]; mean, 0.5019). Session 1 had 720 trials, and we simulated chance performance by taking the mean of 720 random draws from the set [0 1]; we then repeated this procedure 10,000 times to build a distribution of simulated guessing behavior and excluded any participant whose overall accuracy in Session 1 fell within the $95 \%$ confidence interval of this distribution (proportion correct $<=0.5361$ ). The remaining 31 participants form the basis of the unregistered analyses reported below.

Of the 31 above-chance participants, an additional nine observers were excluded from the preregistered analyses 
based on the results of the following eye-tracking analysis: We flagged trials in which an observer's gaze could not be verified to be within $1^{\circ}$ of visual angle (d.v.a.) from the fixation point (either due to a fixation break or because the tracker lost the pupil) during the presentation of the stimuli (see Eye tracking in the online Supplementary Methods), and, only for the purposes of this exclusion analysis, we counted these trials as errors. We then repeated the same simulation of guessing behavior described above, leaving us with 22 above-chance participants. Simply excluding trials in which fixation was unverified (and calculating Session 1 accuracy with the remaining trials) would have led to the inclusion of some participants with very little data (e.g., observers with as few as two, and in one case zero, trials in our primary conditions of interest: Task performance at the previously most-cued and previously least-cued sides).

Chance accuracy in Sessions 2 and 3 was assessed using the same procedure described above, with the exception that only 360 random draws were used (to match the number of trials in each of these sessions). Of the 22 participants, 20 of them met inclusion criteria in Session 2, and 19 of them met the inclusion criteria in Session 3, thus accounting for the changes in the degrees of freedom reported in Supplementary Table 1. Most participants were psychophysically inexperienced undergraduates, and chance performance may indicate a lack of motivation to perform the task and/or an unwillingness to maintain fixation, rather than a genuine lack of understanding or problem with task difficulty.

\section{Additional details}

Please see the online Supplementary Methods for additional methodological details.

\section{Results - preregistered analyses}

In the first half of Session 1, spatially uninformative, exogenous cues reflexively modulated visual processing. Orientation discrimination accuracy was significantly greater when the cue appeared near the location of the forthcoming target (valid trials), relative to when it appeared near the location of the forthcoming distractor (invalid trials): paired t-test, $\mathrm{t}(21)=5.0955, \mathrm{p}<0.0001$; mean within-participant change $=$ 0.0446 , bootstrapped $95 \%$ confidence interval, [0.0278 0.0616 ]; randomization test, $\mathrm{p}<0.0001$ (Fig. 2a). An analogous examination of the response-time (RT) data verified that these changes in accuracy were not due to a speed-accuracy trade-off, as RT significantly decreased on valid, relative to invalid, trials: paired t-test, $\mathrm{t}(21)=-3.8696, \mathrm{p}=0.0009$; mean within-participant change $=-0.0474$ seconds, bootstrapped 95\% confidence interval, [-0.0759- -0.0243$]$; randomization test, $\mathrm{p}=0.0006$ (Fig. 2e). These results confirm that covert exogenous spatial attention was successfully manipulated by the abrupt-onset cues, and because cues appeared twice as often on one side, relative to the other, different histories of exogenous selection were established at the two task locations.

Regarding our primary hypothesis, we found no evidence that a location-specific history of involuntary selection modulated visual processing when abrupt-onset cues were removed in the second half of Session 1. Orientation discrimination accuracies at the previously most-cued and previously least-cued locations were statistically indistinguishable: paired $\mathrm{t}$-test, $\mathrm{t}(21)=-1.0574, \mathrm{p}=0.3023$; mean within-participant change $=-0.0267$, bootstrapped $95 \%$ confidence interval, [-0.0759-0.0210]; randomization test, $p=0.3067$ (Fig. 2b). Additionally, we found no evidence for a selection historybased difference in RT: paired t-test, $\mathrm{t}(21)=1.274, \mathrm{p}=$ 0.2166 ; mean within-participant change $=0.0228 \mathrm{~s}$, bootstrapped $95 \%$ confidence interval, [-0.0132 -0.0557$]$; randomization test, $p=0.2037$ (Fig. 2f). Finally, the magnitude of individual cueing effects was not significantly correlated with the magnitude of selection history effects, for either performance metric: accuracy, $r(21)=0.2384, p=0.2853$, randomization test, $\mathrm{p}=0.2913$; RT: $\mathrm{r}(21)=0.2889, \mathrm{p}=0.1923$, randomization test, $p=0.1914$. Not surprisingly, we also found no evidence for any residual, location-specific selection history effects the following day (Session 2, Fig. 2c and g) or 1 week later (Session 3, Fig. 2d and h): all $p \mathrm{~s}>0.3762$, see Supplementary Table 1 for full reporting.

\section{Results - unregistered analyses}

A major strength of the Registered Report publishing mechanism is that all statistical analyses are pre-planned and reviewed before data collection begins. However, because the experimental outcome itself cannot be known in advance, it is sometimes helpful to follow up the preregistered analyses with additional statistical queries. Below, we present the results of a set of analyses that, while not preregistered, aim to provide more context and a richer depiction of the dataset.

Given this project's focus on spatially-specific exogenous selection history, we investigated the exogenous cueing effect more fully by completing a repeated-measures ANOVA on the accuracy and RT data from the first half of Session 1. We found no evidence for a target side $\mathrm{X}$ cue condition interaction (both $p \mathrm{~s}>0.1044$ ), no evidence for a main effect of target side (both $p \mathrm{~s}>0.2624$ ), and as expected, a significant main effect of cue condition (both $p s<0.001$ ).

Some participants were excluded from the preregistered analyses reported above due to fixation verification concerns (see Data exclusion criteria in Methods). In addition to intentional breaks in fixation, missing eye data can result from a bad initial calibration, excessive post-calibration movement, and/or 


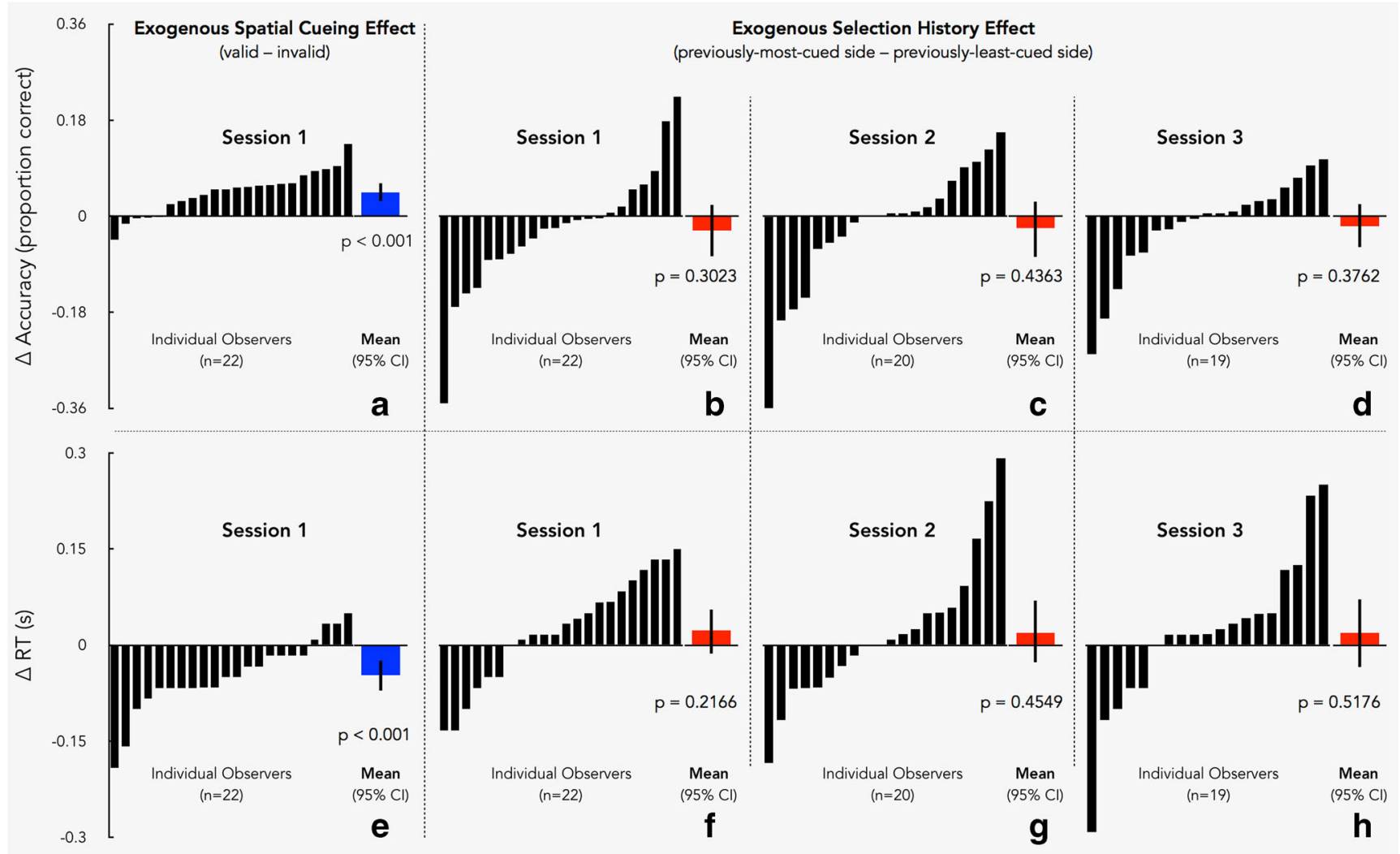

Fig. 2. Results from Preregistered Analyses. Black bars (individual observers), blue and red bars (group means); error bars reflect bootstrapped 95\% confidence intervals. A,E) First half, Session 1. B,F) Second half, Session 1. C,G) Session 2. D,H) Session 3

difficulties in maintaining the pupil because of idiosyncratic features of a participant's eye. Nine additional participants performed above chance and would have been included if we had not taken eye-tracking data into account. For the exogenous cueing results, disregarding eye data should not be a concern, as the target was equally likely to appear at either location and the exogenous cues themselves were spatially uninformative. Furthermore, intentional eye movements toward the previously most-cued side, were they to be present, would only increase the likelihood of observing a significant difference between the two task locations.

Repeating Session 1 analyses with all trials included and with these 31 above-chance participants did not alter the outcome; we again saw significant exogenous cueing effects in the first half, for both accuracy and RT, and when the cues were removed in the second half, there was still no evidence that task performance differed between previously most-cued and previously least-cued sides (see Table 1 for full reporting). This unregistered analysis is presented to alleviate potential concerns about the number of participants excluded from the preregistered analyses and to document that the results are unchanged when all participants who performed above chance in the task are included.

Despite demonstrating ample statistical power to detect exogenous spatial attention effects on both discrimination accuracy and RT, readers may nonetheless be concerned about how to interpret the failure to find evidence in support of our main hypothesis. To provide further treatment of this important issue, we completed a Bayesian statistical approach that allows for quantification of evidence in favor of both the alternative and null hypotheses (JASP Team, 2019; Morey \& Rouder, 2015; Rouder, Speckman, Sun, Morey, \& Iverson, 2009). Conducting Bayesian paired-samples t-tests on exogenous spatial cueing data from our 31 above-chance participants, we found "strong" and "positive" evidence that favored the alternative hypothesis (that exogenous cues modulated task performance) over the null hypothesis (that exogenous cues had no effect) for accuracy and RT, respectively (descriptive labels following Raftery, 1995; Table 1). For the selection history data, we found "positive" and "weak" evidence that favored the null hypothesis (that exogenous selection history had no effect) over the alternative hypothesis for accuracy (our primary dependent variable) and RT, respectively (Table 1). In addition to the selection history data being more likely under the null model, it is also important to note that the mean values of both performance metrics lie opposite to what was predicted by our hypothesis (i.e., accuracy is reduced, and RTs lengthened, on the previously most-cued side). Taken together, we feel confident that the failure to find evidence for the exogenous selection history hypothesis is not due to a lack of statistical power. 
Table 1 Full statistics for Session 1; no trials are excluded and all 31 above-chance participants are included. Significant p-values are in bold

\begin{tabular}{|c|c|c|c|c|}
\hline & \multicolumn{2}{|l|}{ Cue Validity } & \multicolumn{2}{|l|}{ Selection History } \\
\hline & Accuracy & RT & Accuracy & RT \\
\hline t-statistic & 4.0172 & -3.1794 & -0.9415 & 1.2689 \\
\hline df & 30 & 30 & 30 & 30 \\
\hline p-value & 0.0003 & 0.0034 & 0.3540 & 0.2142 \\
\hline mean within-participant change & 0.0321 & -0.0361 & -0.0170 & 0.0207 \\
\hline bootstrapped $95 \%$ confidence interval & {$[0.0167-0.0474]$} & {$[-0.0581--0.0145]$} & {$[-0.0539-0.0167]$} & {$[-0.0110-0.0512]$} \\
\hline randomization test p-value & 0.0004 & 0.0029 & 0.3611 & 0.2088 \\
\hline Bayes factor in support of the null hypothesis & 0.0124 & 0.0893 & 3.4722 & 2.5189 \\
\hline Bayes factor in support of the alternative hypothesis & 80.611 & 11.197 & 0.288 & 0.397 \\
\hline
\end{tabular}

\section{Discussion}

In this Registered Report, we tested the hypothesis that a history of involuntary attentional selection, driven by abruptonset cues that engender the reflexive allocation of spatial attention to cued locations, leads to the formation of spatially-specific selection biases like those that have been observed with voluntarily attended and reward-predictive features (Awh, Belopolsky, \& Theeuwes, 2012; Le Pelley et al., 2016). To build spatially-distinct histories of exogenous selection, we modified the standard cueing paradigm and presented abrupt-onset cues twice as often at one of two task locations. Orientation discrimination accuracy increased, and RTs decreased, when the cue appeared near the location of the forthcoming target, relative to the distractor, thus confirming the successful manipulation of covert exogenous spatial attention. To test our hypothesis, we removed abrupt-onset cues entirely and evaluated task performance at the previously most-cued and previously least cued locations. In short, we found no evidence that a history of involuntary selection generated the kinds of enduring attentional biases that have been observed with a history of goal-directed attentional allocation.

These results suggest potential constraints around the type of selection history that can engender lingering attentional biases, thus advancing our theoretical understanding of selection history's role in attentional control. Below, we discuss three potential interpretations that future research can work to disentangle: 1) a more extensive history of exogenous selection may be necessary, 2) endogenous and exogenous selection histories may be in competition, and 3) selection history effects may rely less on attentional selection and more on the visual processing that typically follows goal-directed attentional allocation. ${ }^{2}$

A history of voluntary selection, for as few as 240 trials, has been shown to be sufficient for generating persistent at-

\footnotetext{
${ }^{2}$ We would like to thank Dr. Edward Awh for this suggestion.
}

tentional biases that interfere with subsequent goal-directed behavior, for both previously reward-predicting (e.g., Anderson \& Halpern, 2017) and never reward-predicting visual features (e.g., Grubb \& Li, 2018). However, it could be the case that a more extensive history than the 360 trials used in this study is necessary for involuntary selection to produce similar effects. Such a history could be derived by lengthening the proportion of the study in which exogenous cues are presented, after which selection history effects could be evaluated at the previously most-cued and previously least-cued locations.

According to the integrated framework of attentional control presented by Awh and colleagues, current goals, selection history, and physical salience all contribute to the construction of an integrated priority map (Awh, Belopolsky, \& Theeuwes, 2012). Because the target in our study was equally likely to appear at one of two known locations, both when exogenous cues were presented (first half) and when the cues were removed (second half), the optimal endogenous strategy would have been to distribute equal amounts of attention across the two locations, thus pitting equivalent histories of voluntary selection at the two locations against unequal histories of involuntary selection. Both exogenous and endogenous selection histories might contribute to the creation of the proposed priority map, but endogenous selection history might be weighted more heavily by the visual system. If this were the case, increasing the number of task locations across which one could voluntarily distribute attention might lessen the competition, thus allowing involuntary selection history to modulate the priority map in a measurable way. Fully characterizing the interaction of endogenous and exogenous spatial attention, in the absence of selection history considerations, required an examination of the full time-course of visual processing (Grubb et al., 2015), and a similar approach might reveal more nuanced findings on exogenous selection history than were observed here.

Finally, visual processing and perceptual action often follow goal-directed attentional allocation. For example, in the 
value-driven attentional capture and accuracy-based feedback follow-up studies mentioned above, colors that reflexively captured attention during the test phase were not only previously attended during training, but participants were also required to process visual information contained within the colored circle and make a perceptual judgment immediately following selection. In the current study, visual attention was involuntarily drawn to a particular spatial location, but because abrupt-onset cues were equally likely to be valid and invalid, perceptual judgments regarding the stimulus at the attended location were only required half of the time. Thus, despite asymmetrical histories of involuntary selection at the two task locations, visual processing in the service of a perceptual action (i.e., the orientation discrimination judgment) was equivalent at the previously-most-cued and previouslyleast-cued locations, which could account for our failure to find a difference in task performance. Anticipatory action can be a powerful regulator of human behavior (Hommel, 2009), and in the voluntary selection history case, anticipatory action is linked directly to attentional selection; this may be a necessary component in the formation of selection historybased effects.

Acknowledgements We would like to thank Dr. Edward Awh and an anonymous reviewer for helpful feedback on the preregistration plan and final manuscript. This study was supported by Trinity College start-up funding to $\mathrm{MG}$.

Author contributions MG designed the study; GC and JA collected data; all authors analyzed data and wrote the manuscript.

\section{Compliance with ethical standards}

Open Practices Statement This study was preregistered, and the data necessary to replicate the reported analyses are available from the corresponding author upon email request.

\section{References}

Anderson, B. A., \& Halpern, M. (2017). On the value-dependence of value-driven attentional capture. Attention, Perception, \& Psychophysics, 79, 1001-1011.

Attention, Perception, \& Psychophysics. (2013). Registered reports and replications in Attention, Perception, \& Psychophysics. Attention, Perception, \& Psychophysics, 75, 781.

Awh, E., Belopolsky, A. V., \& Theeuwe s, J. (2012). Top-down and bottom-up attentional control: A failed theoretical dichotomy. Trends in Cognitive Sciences, 16, 437-443.

Cameron, E. L., Tai, J. C., \& Carrasco, M. (2002). Covert attention affects the psychometric function of contrast sensitivity. Vision Research, 42, 949- 967.

Carrasco, M. (2011). Visual attention: The past 25 years. Vision Research, $51,1484-1525$.
Carrasco, M., Ling, S., \& Read, S. (2004). Attention alters appearance. Nature Neuroscience, 7, 308-313.

Carrasco, M., \& McElree, B. (2001). Covert attention accelerates the rate of visual information process- ing. Proceedings of the National Academy of Sciences, USA, 98, 5363-5367.

Giordano, A. M., McElree, B., \& Carrasco, M. (2009). On the automaticity and flexibility of covert attention: A speed-accuracy trade-off analysis. Journal of Vision, 9(3):30, 1-10.

Grubb, M. A., Behrmann, M., Egan, R., Minshew, N. J., Heeger, D. J., \& Carrasco, M. (2013). Exogenous Spatial Attention: Evidence for Intact Functioning in Adults With Autism Spectrum Disorder. Journal of Vision, 13(14): 9, 1-13.

Grubb, M. A., \& Li, Y. (2018). Assessing the role of accuracy-based feedback in value-driven attentional capture. Attention, Perception, and Psychophysics, 80: 822-828.

Grubb, M. A., White, A. L., Heeger, D. J., \& Carrasco, M. (2015). Interactions between voluntary and involuntary attention modulate the quality and temporal dynamics of visual processing. Psychonomic Bulletin \& Review, 22, 437-444.

Herrmann, K., Montaser-Kouhsari, L., Carrasco, M., \& Heeger, D. J. (2010). When size matters: Attention affects performance by contrast or response gain. Nature Neuroscience, 13, 1554-1559.

Hommel, B. (2009). Action control according to TEC (theory of event coding). Psychological Research PRPF, 73(4), 512-526.

Hopfinger, J. B., \& Mangun, G. R. (1998). Reflexive attention modulates processing of visual stimuli in human extrastriate cortex. Psychological Science, 9, 441-447.

JASP Team (2019). JASP(Version 0.8.6)[Computer software].

Jiang, Y. V. (2017). Habitual versus goal-driven attention. Cortex, https:// doi.org/10.1016/j.cortex.2017.06.018

Le Pelley, M. E., Mitchell, C. J., Beesley, T., George, D. N., \& Willis, A. J. (2016). Attention and associative learning in humans: An integrative review. Psychological Bulletin, 142, 1111-1140.

Ling, S., \& Carrasco, M. (2006). When sustained attention impairs perception. Nature Neuroscience, 9(10), 1243-1245.

Liu, T., Pestilli, F., \& Carrasco, M. (2005). Transient attention enhances perceptual performance and fMRI response in human visual cortex. Neuron, 45, 469-477.

Morey, R. D., \& Rouder, J. N. (2015). BayesFactor (Version 0.9.113)[Computer software].

Muller, H. J., \& Rabbitt, P. M. (1989). Reflexive and voluntary orienting of visual attention: time course of activation and resistance to interruption. Journal of Experimental Psychology: Human Perception and Performance, 15(2), 315-330.

Nakayama, K., \& Mackeben, M. (1989). Sustained and transient components of focal visual attention. Vision Research, 29(11), 1631-1647.

Raftery, A. E. (1995). Bayesian model selection in social research. In P. V. Marsden (Ed.), Sociological methodology 1995 (pp. 111-196). Cambridge, MA: Blackwell.

Rouder, J. N., Speckman, P. L., Sun, D., Morey, R. D., \& Iverson, G. (2009). Bayesian $t$ tests for accepting and rejecting the null hypothesis. Psychonomic Bulletin \& Review, 16, 225-237.

Yeshurun, Y., \& Carrasco, M. (1998). Attention improves or impairs visual performance by enhancing spatial resolution. Nature, 396, $72-75$.

Publisher's note Springer Nature remains neutral with regard to jurisdictional claims in published maps and institutional affiliations. 